\title{
TERMINAL
}

\section{Externe Prognosegutachten \\ Fragenkatalog der Göttinger Strafvollstreckungskammer an einen psychiatrischen Sachverständigen}

In Anlehnung an die: "Checklist of Points Considered by the Home Office in Examining the Cases of Restricted Patients«, abgedruckt bei: Sara Dent (1997), The Home Office Mental Health Unit and its approach to the assessment and management of risk, in: International Review of Psychiatry (1997), 9, 265 - 271.

\section{Generelle Items}

1. Lag den Anlasstaten des Verurteilten aus heutiger, rückwirkender Sicht eine seelische Störung im Sinne der Voraussetzungen des $\S 20$ StGB zugrunde? Wenn ja: Wie ist diese Störung diagnostisch einzuordnen? Welche Auswirkungen hatte diese Störung auf die Schuldfähigkeit des Verurteilten (aufgehobene oder erheblich verminderte Einsichts- oder Steuerungsfähigkeit, §§ 20, 21 StGB)? Welche Auswirkungen hatte diese Störung auf die Gefährlichkeit des Verurteilten ( $(63$ StGB)?

2. Ist der Verurteilte schon im Vorfeld der Anlasstaten durch rechtswidrige Taten aufgefallen, die auf eine seelische Störung zurückgehen? Bejahendenfalls: Um welcher Art seelische Störung handelte es sich? Welche Auswirkungen hatte diese Störung auf die Schuldfähigkeit (\$§ 20, 21 StGB) des Verurteilten bei Begehung dieser Taten? Welche Auswirkungen hatte sie auf die Gefährlichkeit des Verurteilten (§ $63 \mathrm{StGB})$ ? Sind schon im Vorfeld der Anlasstaten sonst Verhaltensauffälligkeiten des Verurteilten hervorgetreten, die im Zusammenhang mit einer seelischen Störung stehen?

3. Welche Veränderung hat die seelische Störung im Verlaufe der bisherigen Maßregelbehandlung erfahren und wie wirkt sich diese Veränderung auf die Einschätzung der Gefährlichkeit des Verurteilten aus?

4. Worin äußert sich diese Veränderung, z. B. im Verhalten gegenüber Mitpatienten und Behandlern im Vollzug der Unterbringung oder bei Lockerungen? Insbesondere: Hat es im Unterbringungsverlauf besondere Belastungssituationen gegeben und wie ist der Verurteilte damit umgegangen? Hat er körperliche Gewaltbereitschaft oder verbale Aggressivität gezeigt?

5. Worin könnten aus heutiger Sicht für die Zukunft weiterhin relevante potentielle Risikofaktoren liegen? Wie könnten diese Risikofaktoren in ihrer Bedeutung abgemildert oder gar vollständig aufgefangen werden?

6. Welche Einstellung hat der Verurteilte heute zu seinen Anlasstaten und zu seinen Opfern?

7. Hat der Verurteilte in der Vergangenheit Alkohol oder illegale Drogen konsumiert und hat deren Konsum zu den Anlasstaten beigetragen? Bejahendenfalls: Besteht eine Alkohol- oder Drogenproblematik im Rahmen der Unterbringung fort? Wie ist die gegenwärtige Einstellung des Verurteilten zu Alkohol und Drogen? Sind spezifische Behandlungsversuche der Substanzmissbrauchsproblematik unternommen worden?

8. Welche Aspekte müssen noch ärztlich / therapeutisch bearbeitet werden? Welche kurz-, mittel- oder langfristigen Behandlungsmöglichkeiten stehen insoweit zur Verfügung und welche dieser Möglichkeiten erscheinen dabei im konkreten Fall vorzugswürdig?

\section{Seelisch-kranke Verurteilte, insbesondere Schizophrene}

9. Wie verhält sich die Gefährlichkeit des Verurteilten zu seiner geistigen Erkrankung? Inwiefern ist die Gefährlichkeit für die Erkrankung symptomatisch?

10. Welche Symptome der Erkrankung bestehen fort?
11. Konnte die psychische Stabilität des Verurteilten unter verschiedenen, wechselnden Bedingungen aufrechterhalten werden? Unter welchen Bedingungen könnte die psychische Stabilität gefährdet sein?

12. Haben Medikamente geholfen? Wie wichtig sind sie für die Gewährleistung der psychischen Stabilität des Verurteilten?

13. Hat der Verurteilte Einsicht in seine Krankheit und die Notwendigkeit ihrer medikamentösen Behandlung, gegebenenfalls in welchem Maße?

14. Hat sich der Verurteilte während seiner Unterbringung mit seiner medikamentösen Behandlung einverstanden gezeigt? Hat er Vorbehalte gegenüber der medikamentösen Behandlung erkennen lassen? Würde er die medikamentöse Behandlung außerhalb der stationären Unterbringung fortsetzen?

\section{Persönlichkeitsgestörte Verurteilte}

15. Welche individuellen Charakteristika weist die Persönlichkeitsstörung des Verurteilten auf?

16. Welche spezifischen Störungsbereiche sind im Verlaufe der bisherigen Behandlung angegangen worden? Mit welchem Erfolg?

17. Erscheint der Verurteilte heute in seinem Verhalten gereifter und verlässlicher und mehr für die Belange anderer aufgeschlossen? Woraus ist das ablesbar?

18. Verfügt der Verurteilte heute über eine größere Frustrations- und Stresstoleranz? Woraus ist das ablesbar?

19. Ist der Verurteilte heute in besser in der Lage, die Folgen seines Handelns abzuschätzen und aus Erfahrungen zu lernen, gegebenenfalls in welchem Maße? Woraus ist das abzuleiten?

\section{Minderbegabte Verurteilte}

21. Hat der Verurteilte von seiner Behandlung und den bisher durchgeführten sozial- und verhaltenstherapeutischen Maßnahmen profitiert? Woraus ergibt sich das?

22. Erscheint das Verhalten des Verurteilten heute adäquater, gegebenenfalls inwiefern?

23. Reagiert der Verurteilte explosiv oder handelt er impulsiv? Woraus ergibt sich das? Ist der Verurteilte heute in der Lage, aus Erfahrung zu lernen und die Folgen seines Verhaltens abzuschätzen? Woraus ergibt sich das?

\section{Sexualdelinquenten}

24. Zeigt der Verurteilte weiterhin ein inadäquates Interesse für seinen Opfertyp (z. B. Kinder bestimmter Altersgruppen oder bestimmten Geschlechts, Frauen oder Männer bestimmter Altersgruppen oder mit bestimmten sonstigen besonderen Merkmalen)? Hat sich seine Einstellung insoweit verändert, gegebenenfalls inwiefern? Woraus ist das ablesbar?

25. Hatte der Verurteilte während seiner bisherigen Unterbringung Gelegenheit, seinem Opfertyp zu begegnen? Bejahendenfalls: Wie hat er sich verhalten?

26. Zeigt der Verurteilte weiterhin ein Vorliebe für bestimmte Praktiken (z. B. bestimmte Sexualtechniken, Exhibitionismus, Fetischismus, sadistische oder masochistische Prak- tiken, allgemein Anwendung von Gewalt)? Hat sich seine Einstellung insoweit verändert, gegebenenfalls inwiefern? Woraus ist das ablesbar?

27. Hatte der Verurteilte während seiner bisherigen Unterbringung Gelegenheit zu sexuellen Aktivitäten, gegebenenfalls welcher Art?

28. Welchen Inhalt haben die sexuellen Phantasien des Verurteilten heute?

\section{Brandstifter}

29. Interessiert sich der Verurteilte noch für Feuer, gegebenenfalls inwiefern?

30. Hat der Verurteilte während seiner Unterbringung Feuer gelegt oder dieses versucht?

31. Welchen Zugang hat der Verurteilte zu Feuerzeugen und Streichhölzern?

32. Hat der Verurteilte Phantasien, die Feuer zum Gegenstand haben? Wenn ja, welcher Art sind diese Phantasien?

33. Zeigt der Verurteilte Einsicht in sein früheres Verhalten als Brandstifter und in die Ursachen dieses Verhaltens?

\section{Allgemeines}

34. Zu welchen Ergebnissen haben psychologische Testungen und sonstige, insbesondere neurologische oder radiologische Untersuchungen geführt und welche Bedeutung haben diese Ergebnisse für die diagnostische Einordnung des Störungsbildes und die Prognose?

35. Welche weiteren Aspekte oder Faktoren erscheinen prognostisch bedeutsam?

\section{Schlussfolgerungen}

36. Mit welchem Maß an Wahrscheinlichkeit kann nach alledem bereits jetzt angenommen werden,

- dass bei dem Verurteilten keine Gefahr mehr besteht, dass dessen durch seine Taten zutage getretene $\mathrm{Ge}$ fährlichkeit fortbesteht (§§ 454 Abs. 2, 463 Abs. 3 StPO) und mit welchem Maß an Wahrscheinlichkeit ist zu erwarten,

- dass der Verurteilte außerhalb des Maßregel- und Strafvollzuges keine rechtswidrigen Taten mehr begehen wird ( $\$ 67 \mathrm{~d}$ Abs. 2 StGB)?

37. Durch welche begleitenden und stützenden Maßnahmen (Auflagen oder Weisungen) könnte die Wahrscheinlichkeit straffreier Führung außerhalb des Maßregel- und Strafvollzuges abgesichert oder gar weiter erhöht werden?

38. Bedarf der Verurteilte derzeit noch weiterer stationärer Behandlung im Rahmen der Unterbringung in einem psychiatrischen Krankenhaus gemäß § 63 StCB?

39. Bejahendenfalls: Welcher Sicherungsgrad erscheint erforderlich (geschlossene, halboffene, offene Unterbringung; begleiteter, unbegleiteter Gemeinschafts- oder Einzelausgang, Beurlaubungen)?

40. Auf welche Weise und mit welchen Maßnahmen kann und sollte der Verurteilte durch die weitere Maßregelunterbringung weiter gefördert werden? 